\title{
ALGUNAS CARACTERÍSTICAS DE LA FECUNDIDAD RURAL EN MÉXICO
}

\author{
JULIETA QUILODRÁN \\ El Colegio de México
}

\section{INTRODUCCIÓN}

EL PROPÓSITO DE este trabajo es caracterizar, a grandes rasgos, el tipo de fecundidad que impera entre las mujeres residentes en el área rural de México. La información de la cual se desprenden los índices que van a servir para definir el régimen de fecundidad predominante, provienen de la Encuesta de Fecundidad Rural (PECFAL-R) llevada a cabo en México a fines de 1969 y principios de 1970, como parte del Programa de Encuestas Comparativas de Fecundidad en América Latina (PECFAL).

En realidad, el objetivo es demostrar que la población rural de México no controla su fecundidad y si lo hace, los métodos que utiliza no son eficaces. Esta demostración exige el cálculo de una serie de tasas y otros indicadores que tienen importancia en sí mismos, ya que van a reflejar de manera bastante precisa, niveles y diferenciales de fecundidad en la población estudiada. Dada la magnitud de los índices de fecundidad que se conocen para México, es poco probable que llegue a comprobarse que el sector rural del país esté limitando sus nacimientos.

A través de las preguntas del cuestionario de la encuesta referidas a la fecha de nacimiento de la mujer entrevistada y la fecha de nacimiento de cada uno de los hijos nacidos vivos (historia de embarazos), se pudo establecer la edad de la madre al tener cada uno de ellos. La obtención de este dato permitió establecer la distribución de las mujeres que se encontraban unidas en el momento de la encuesta o lo habían estado con anterioridad, según la edad al tener cada uno de los hijos nacidos vivos, el orden de nacimiento de éstos, la edad en la primera unión y el grupo de generaciones al que pertenecen. De aquí en adelante, para mayor facilidad, se denominará "mujeres alguna vez unidas" al grupo de mujeres cuyo estado civil era diferente del de "soltera" en el momento de la encuesta.

Tabulaciones de la misma naturaleza que las mencionadas ofrecen ma- 
yores posibilidades de análisis que los realizados en este trabajo. Ellas permiten calcular, para el conjunto de mujeres clasificadas por grupos de generaciones, los intervalos intergenésicos entre hijos nacidos vivos, el calendario de los nacimientos, la edad promedio de las madres al tener sus hijos según el orden de los nacimientos, descendencias finales o hasta la edad x, para mencionar sólo algunos ejemplos. Sin embargo, el objetivo planteado en este trabajo, así como la elaboración reciente de estas tabulaciones, han limitado su utilización.

Se puede caracterizar la fecundidad recurriendo a diversos tipos de medidas, según se hagan intervenir o no variables tales como: la edad de la mujer, su estado civil, su edad en la primera unión, la duración de ésta o de otras uniones; ya sea que el uso de las mismas se haga en forma individual, combinadas entre sí o con otras variables apropiadas a los fines que se persiguen. Estudios realizados sobre la fecundidad de diferentes tipos de poblaciones ${ }^{1}$ han demostrado que en aquéllas donde las mujeres no ejercen control sobre su fecundidad, las tasas de fecundidad legítimas (legales) según los grupos de edad, varían en función de la edad de la mujer; o sea, que dentro de un grupo de edades determinado las tasas de fecundidad son muy similares, independientemente del tiempo que haya transcurrido desde el inicio de la unión. El hecho que las tasas de fecundidad de las mujeres que iniciaron su primera unión en edades diferentes resulten semejantes a las tasas de fecundidad de grupo de edades determinado cuando se considera la edad en que tuvieron los hijos, quiere decir que dichas mujeres no controlan su fecundidad; de lo contrario, el calendario y el número de nacimientos de un mismo orden se verían afectados e inmediatamente se traducirían en cambios en los niveles de las tasas correspondientes a un mismo grupo de edad de las mujeres.

Sin embargo, como no se puede definir a una población natural por medio de una serie de tasas solamente, ya que de una población natural a otra estas tasas pueden variar, ${ }^{2}$ resulta insuficiente calcular las tasas de fecundidad correspondientes a la población que se desea caracterizar y compararlas con las tasas de una población que haya sido definida como natural. Para mayor seguridad conviene recurrir a un segundo tipo de medición, que consiste en controlar la edad que tenían la primera vez que se unieron entre aquellas mujeres que hayan completado su descendencia o estén por hacerlo. De esta forma no se mezclan en una misma tasa, hijos de mujeres que mantienen uniones de diferente duración.

Una tercera forma de comprobar una inexistente limitación de nacimientos, consiste en calcular las probabilidades de crecimiento de las familias, calculando la probabilidad que tiene una mujer que posee un hijo de orden i de llegar a tener un hijo de orden $\mathrm{i}+1$. La proporción de mujeres con "al menos un hijo de orden i" pertenecientes a una misma cohorte de mujeres, descenderá más rápidamente en una población que limita los

1 Henry, Louis, 'Demographie, Analyse et Modeles' París, Larousse, 1972.

2 Henry, op. cit. 
nacimientos, puesto que en estas poblaciones una vez alcanzado el número deseado de hijos, la probabilidad de tener otros hijos se reduce. En una población con un régimen de fecundidad "natural" las probabilidades de tener un hijo más, están, como se sabe, ligadas principalmente a la esterilidad involuntaria de las mujeres adquirida a lo largo de su vida reproductiva.

Los datos obtenidos en la encuesta PECFAL-R de México permitieron efectuar mediciones de los tres tipos descritos anteriormente, a saber, tasas de fecundidad por grupos de edades y generaciones para mujeres "alguna vez unidas", tasas de fecundidad según los grupos de edad para mujeres al final de su vida reproductiva de acuerdo con la edad en la primera unión y probabilidades de crecimiento de las familias. A continuación se presenta la forma en que fueron elaborados estos datos y el análisis que con ellos se realizó.

La población considerada en este trabajo está compuesta por mujeres con una o más uniones de naturaleza legal o libre (convivencia) interrumpidas o subsistentes en el momento de la entrevista. El hecho de incluir mujeres con uniones libres no ofrece problemas en tanto estas uniones sean lo suficientemente estables. ${ }^{3}$ Ahora bien, las mujeres con uniones interrumpidas representan el $9.2 \%$ del total de mujeres "alguna vez unidas" y el $5 \%$ del total de mujeres "alguna vez unidas" de 35 años y más, grupo sobre el cual se calcularon las tasas de fecundidad según edad en la primera unión y las probabilidades de crecimiento de las familias. El hecho de que estas mujeres con uniones interrumpidas, se distribuyan según su edad en la primera unión de la misma forma que las mujeres que se encuentran unidas en el momento de la encuesta, asegura que los niveles de la fecundidad no se verán alterados en función de esta característica.

Del total de mujeres "alguna vez unidas", el $22.1 \%$ está constituido por mujeres que se encuentran en unión libre o son viudas o están separadas de una unión de este tipo, de modo que su exclusión habría disminuido bastante el número de casos por considerar. ${ }^{4}$ El mayor problema que ofrece la unión libre en el caso de las mujeres analizadas, consiste en que entre ellas la proporción de separadas es más elevada que entre las mujeres unidas legalmente; pero si se considera el hecho de que la proporción de las mujeres con una sola unión es de alrededor del $90 \%$, la influencia de las separaciones sobre la fecundidad debería ser muy reducida. ${ }^{5}$

Estas consideraciones, aunadas al número relativamente bajo de observaciones, determinó que se tratara al conjunto de las mujeres como si

\footnotetext{
3 Datos de esta misma encuesta arrojan para el conjunto de mujeres con una o más uniones libres, una duración total de las uniones de 25.4 años al final de su vida reproductiva, en comparación con los 27.1 años de las mujeres que estuvieron siempre unidas legalmente.

4 Julieta Quilodrán de Aguirre. "Nupcialidad y Fecundidad en la Encuesta de Fecundidad Rural en México" (trabajo mimeografiado), cuadro 3, p. 17.

5 Incluyendo las mujeres que contraen, con el hombe con quien convivían, una segunda unión de tipo legal.
} 
fuera uno solo, cualquiera que fuera la naturaleza de la unión y el número de uniones, fueran éstas subsistentes o no.

Las medidas de fecundidad que surgidas de los tipos de medición antes señalados, deberían estar exentas de influencias de fenómenos perturbadores, tales como la mortalidad y la migración, con el fin de que los índices que se elaboren reflejen lo que serían los niveles de la fecundidad en "estado puro". Como no existen datos que permitan corregir el efecto de estos fenómenos perturbadores, se formula la siguiente hipótesis: "las personas que murieron, de haber sobrevivido, o las que migraron, de haberse quedado, habrían tenido la misma fecundidad que las sobrevivientes y las que no migraron".

Por otra parte, tratándose de un fenómeno renovable como es la fecundidad, se requiere que la hipótesis referida a la influencia de los fenómenos perturbadores tenga vigencia para cada una de las etapas sucesivas de la vida reproductiva de la mujer consideradas en este trabajo.

Teniendo en cuenta estas hipótesis, se buscó establecer distribuciones de la fecundidad de acuerdo con los grupos de edades, con el fin de observar cambios que hubieran podido producirse en el tiempo, $y$, hasta donde fue posible, calcular también indicadores de intensidad como son el número promedio de hijos nacidos vivos y descendencias finales.

\section{Fecundidad por grupos de generaciones}

En la encuesta fueron interrogadas mujeres entre los 15 y los 49 años cumplidos lo que significa en términos de generaciones, mujeres nacidas entre 1920 y 1954 inclusive.

\section{Cuadro 1}

TASAS DE FECUNDIDAD POR GRUPOS DE EDADES Y GENERACIONES, PARA 1000 MUJERES ALGUNA VEZ UNIDAS

\begin{tabular}{lcccccccc}
\hline \multirow{2}{*}{$\begin{array}{l}\text { Edad a tener los } \\
\text { hijos nacidos vivos }\end{array}$} & \begin{tabular}{c} 
Generación \\
\cline { 3 - 8 }
\end{tabular} & $\begin{array}{c}1950-54 \\
15-19\end{array}$ & $\begin{array}{c}1945-49 \\
20-24\end{array}$ & $\begin{array}{c}1940-44 \\
25-29\end{array}$ & $\begin{array}{c}1935-39 \\
30-34\end{array}$ & $\begin{array}{c}1930-34 \\
35-39\end{array}$ & $\begin{array}{c}1925-29 \\
40-44\end{array}$ & $\begin{array}{c}1920-24 \\
45-49\end{array}$ \\
\hline $15-19$ & 250 & 256 & 237 & 258 & 244 & 261 & 230 \\
$20-24$ & & 401 & 408 & 410 & 385 & 406 & 346 \\
$25-29$ & & & 404 & 425 & 399 & 408 & 372 \\
$30-34$ & & & & 364 & 375 & 350 & 320 \\
$35-39$ & & & & 330 & 314 & 256 \\
$40-44$ & & & & & 132 & 147 \\
$45-49$ & & & & & & \\
\hline
\end{tabular}

Las tasas del cuadro 1 se calcularon refiriendo los bijos nacidos vivos que tuvieron las mujeres mientras transitaban por cierto grupo de edades, -y que pertenecían a un mismo grupo de generaciones-, al número de años transcurridos en unión matrimonial dentro del grupo de edades considerando el total de mujeres que se unieron hasta la edad correspondiente 
al límite superior del grupo de edades estudiado (edad en años cumplidos). Ejemplo:

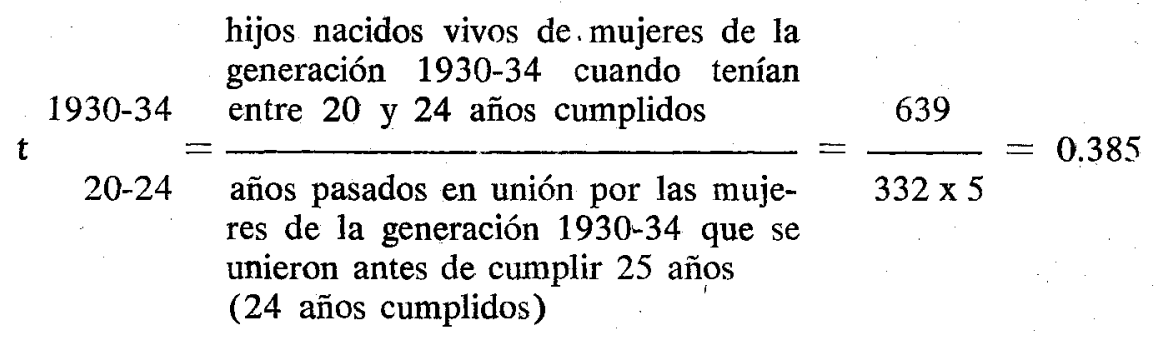

\section{Figura 1}

TASAS DE FECUNDIDAD DE LAS MUJERES "Alguna VeZ UNIDAS" DE LA GENERACIÓN, 1930-1934

(35-39 años al momento de la encuesta)

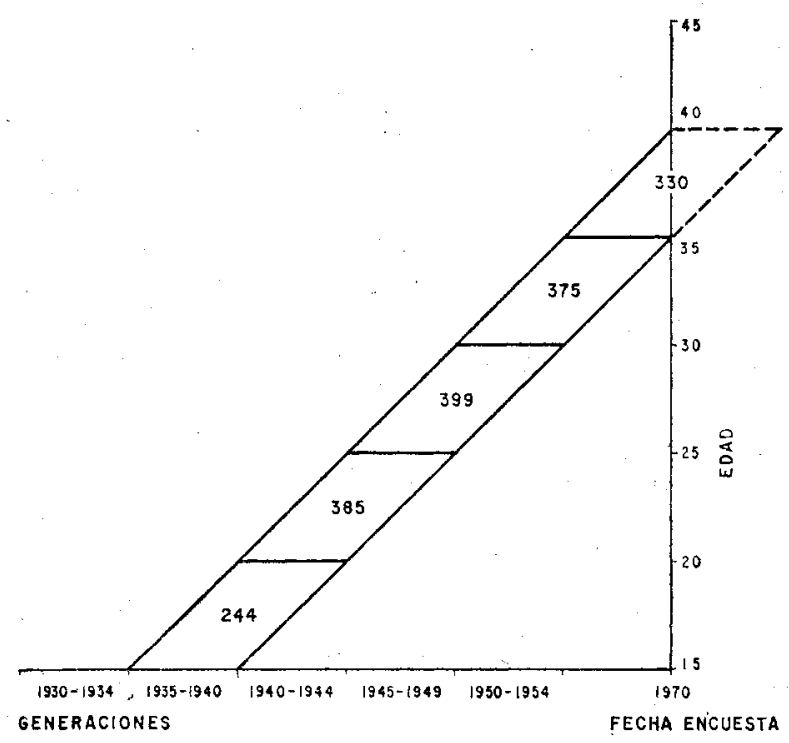

En fecha próxima, se prevé efectuar un cálculo más correcto que consiste en referir los hijos nacidos vivos de cada grupo de edades, al número exacto de años pasados por las mujeres en unión, dentro de ese mismo grupo; o sea, tener en cuenta que el inicio de las uniones no se reparte uniformemente en el interior de cada grupo de edades y que, por lo tanto, el tiempo de permanencia en ese grupo no es igual para todas 
las mujeres. El efecto de la forma de cálculo utilizada (suponer que todas las mujeres dentro de un grupo de edad se unieron al ingresar a él), determina la subestimación de las tasas especialmente en el grupo de edades 15-19 y 20-24 en los que se concentra la celebración de las uniones.

Por otra parte, dada la naturaleza retrospectiva de la información, las mujeres pertenecientes al grupo de edades más avanzado de cada generación no habían recorrido completamente dicho grupo al momento de la encuesta. Teniendo en cuenta este hecho, las tasas correspondientes a estos grupos de edades se estimaron a partir de las tasas que se obtuvieron para las mismas edades en generaciones más antiguas.

En la gráfica 1, donde se representaron las tasas por grupos de generaciones, se observa que en todas las generaciones (salvo para las generaciones 1950-54 en que sólo hay una observación), las tasas aumentan entre los grupos 15-19 y 20-24, alcanzando su máximo alrededor de los 25 años; luego descienden paulatinamente hacia los 40 años y rápidamente después. El pronunciado ascenso que se da entre el primero y segundo grupo de edades debe ser interpretado, en parte, como producto de la subestimación que se habría hecho de la tasa 15-19 años, debido a la forma de calcularla; y en parte también, al incremento de la fertilidad de las mujeres en los primeros años de su vida reproductiva. Para todas las generaciones el periodo más fértil se ubica entre los 20 y los 30 años.

Es notoria, sin embargo, la diferencia que presenta la curva correspondiente al grupo de generaciones 1920-1924; su forma no difiere de las demás, pero los niveles de las tasas son más bajos especialmente en las edades en que la fecundidad suele alcanzar su máximo (alrededor de los 22.5 y 32.5 años). Las tasas de los grupos de edades 15-19 años y 35 y más del grupo de generaciones 1920-1924, se aproximan bastante a los niveles alcanzados por los otros grupos de generaciones en esas mismas edades. Si las tasas extremas no difieren, el hecho de que las tasas para los grupos de edades intermedios (20-24 . . 35-39 años) sean más bajas puede ser atribuido más a omisiones en la declaración del número de hijos nacidos vivos por parte de las mujeres, que a una fecundidad efectivamente más baja entre ellas.

Los hijos de las mujeres de las generaciones 1920-1924 que nacieron cuando ellas tenían entre 22.5 y 37.5 años, lo hicieron entre 1942 y 1957 aproximadamente. En ese período se dio un fuerte descenso de la mortalidad en el país pero perduraban aún niveles elevados. El corto período de supervivencia de muchos niños como resultado de esta generalizada alta mortalidad, podría ser el origen de las omisiones de muchas madres al deciarar el número de hijos nacidos vivos.

Hecha la salvedad relativa a la generación 1920-1924, se puede afirmar que para el resto de las generaciones las tasas de fecundidad para un mismo grupo de edades no difieren mayormente entre sí. 


\section{Gráfica 1}

TASAS DE FECUNDIDAD POR GRUPO DE EDADES Y GENERACIONES, PARA 1000 MUJERES "Alguna VEZ UNIDAS"

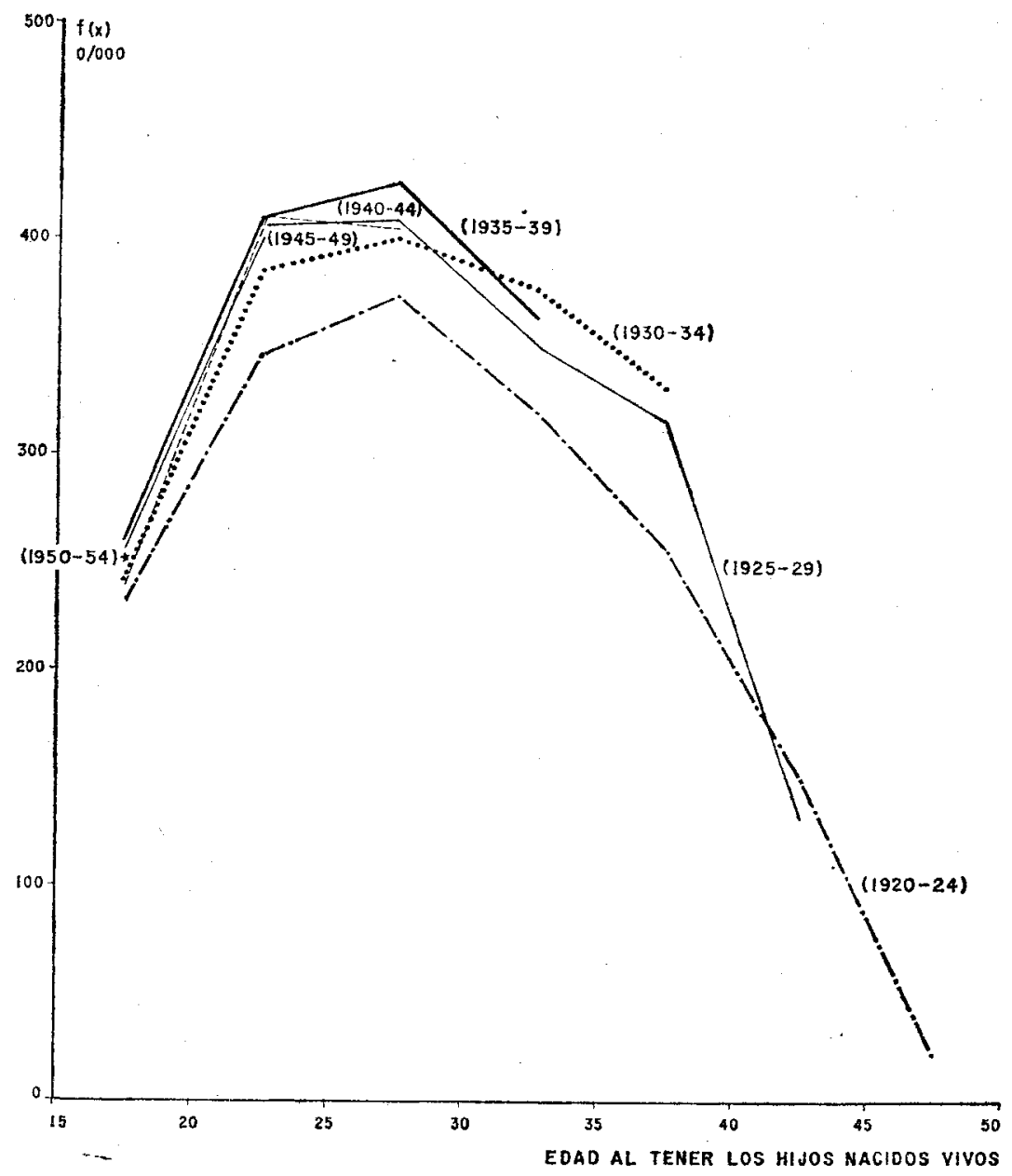

III. Fecundidad Según la edad en la primera unión

Para efectuar la medición de la fecundidad tomando como punto de referencia la edad de las mujeres en la primera unión, se requieren los datos sobre el número de hijos nacidos vivos, la edad que tenían cuando el nacimiento, para aquellas mujeres que hubieran terminado de constituir su descendencia; es decir, mujeres de 45 años y más.

En vista de que el número de mujeres 45-49 años en la encuesta no es 
lo suficientemente numeroso como para obtener tasas por grupos de edades, para cada grupo de edad en el momento del matrimonio, se optó por reunir a todas las mujeres de 35 años y más. Esta asimilación es válida, en la medida que las tasas de fecundidad de los 35 años en adelante son lo bastante bajas como para no afectar significativamente las descendencias finales que se lleguen a obtener. ${ }^{6}$

El cuadro 2, contiene las tasas por grupos de edades de las mujeres que, al tener sus hijos nacidos vivos tenían, en el momento de la encuesta, entre 35 y 49 años cumplidos. Estos datos proporcionan una medida del comportamiento de las mujeres frente a la fecundidad, según haya estado comprendida su edad inicial al unirse, dentro de la generación de 1920-1934.

\section{Cuadro 2}

TASAS DE FeCundidad para 1000 mujeres de 35 a 49 años

"ALGUNA VEZ UNIDAS", SEGÚN EDAD EN LA PRIMERA UNIÓN Y AL TENER LOS HIJOS NACIDOS VIVOS*

\begin{tabular}{|c|c|c|c|c|c|c|c|c|c|c|}
\hline \multirow{2}{*}{$\begin{array}{l}\text { Edad } \\
\text { primera } \\
\text { unión }\end{array}$} & \multicolumn{8}{|c|}{ Edad al tencr los hijos nacicos vivos } & \multirow{2}{*}{$\begin{array}{l}\text { Numero } \\
\text { de } \\
\text { nujeres ** }\end{array}$} & \multirow{2}{*}{$\begin{array}{l}\text { Desce: } \\
\text { denci } \\
\text { medie }\end{array}$} \\
\hline & -15 & $15-19$ & $20-24$ & $25-29$ & $30-34$ & $35-39$ & $40-44$ & $45-49$ & & \\
\hline-15 & 204 & 371 & 353 & 344 & 298 & 217 & 102 & 37 & 129 & 8.83 \\
\hline $15-19$ & & 361 & 431 & 369 & 335 & 215 & 122 & 21 & 446 & 8.48 \\
\hline $20-24$ & & & 409 & 437 & 337 & 238 & 125 & 28 & 211 & 7.05 \\
\hline $25-29$ & & & & 456 & 355 & 189 & 118 & 30 & 53 & 4.91 \\
\hline $30-34$ & & & & & 309 & 267 & 107 & 21 & 15 & 3.02 \\
\hline
\end{tabular}

* Se estimó el número de HNV que faltaban nacer, de las mujeres de 35 a 49 años, en el supuesto de que todas estas generaciones contribuirían con una misma proporción de HNV, en los grupos de edades que les falta por recorrer, que aquellas que ya lo habrán hecho.

** Total de mujeres alguna vez unidas de 35 a 49 años cumplidos $=861$.

La gráfica 2 refleja el incremento de las tasas de fecundidad en los primeros años de unión en aquellos grupos de mujeres que se casaron más jóvenes. A partir de los 30 años comienza el descenso de las tasas que se vuelven prácticamente nulas en el grupo 45-49 años.

El grupo de mujeres que se unió antes de los 15 años, incrementa fuertemente su fecundidad en los primeros años de unión. Este hecho concuerda con el aumento de la fertilidad que se da desde la pubertad hasta alrededor de los 20 años. Su tasa en el grupo 15-19 años es muy semejante a la tasa que presentan para esa misma edad las mujeres unidas entre los 15-19 años; sin embargo, entre los 20 y los 35 años, el nivel de las tasas se ubica por debajo del correspondiente a los otros grupos de edades al casarse, para un mismo grupo de edades, al tener los hijos nacidos vivos. Estos niveles más bajos pueden ser atribuidos en parte, al peso que

${ }^{6} \mathrm{La}$ proporción de HNV que faltarían por nacer entre las mujeres de 35 a 49 años cumplidos, sería de un $9.1 \%$ tomando como base las tasas del cuadro 1 . 


\section{Gráfica 2}

TASAS DE FECUNDIDAd PARA 1000 MUJERES DE 35 A 49 aÑos

"ALGUNA VEZ UNIDAS", SEGÚN EDAD EN LA PRIMERA UNIÓN

$Y$ AL TENER LOS HIJOS NACIDOS VIVOS

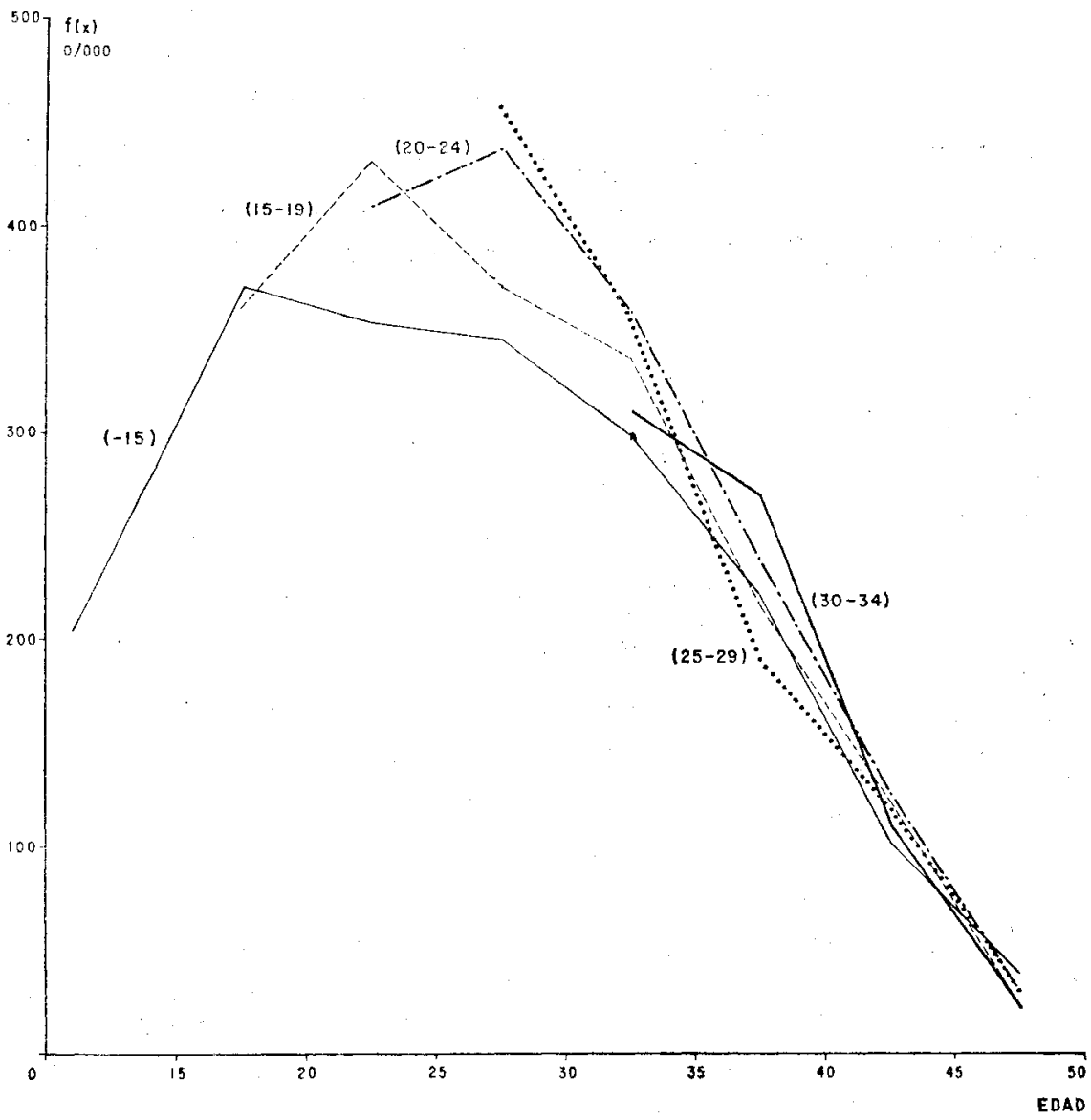

tiene la fecundidad de las mujeres de $45-49$ años, que representan el $28 \%$ del grupo de mujeres de 35-49 años unidas antes de los 15 años. Como se vio al analizar la fecundidad para el conjunto de mujeres "alguna vez unidas", este grupo presenta niveles más bajos de fecundidad que las generaciones más jóvenes. También podrían explicarse por una propensión mayor a la ruptura de uniones entre las mujeres unidas más jóvenes. Esta segunda explicación concordaría con el hecho de que en los primeros años de unión presentan niveles de fecundidad semejantes a los de los otros grupos de edad en la primera unión, pero no con el hecho de que sean de 
nuevo semejantes hacia el final de la vida reproductiva de la mujer. Este hecho puede ser atribuido a una combinación de la deficiente declaración de fechas de nacimiento de los hijos, con la omisión de muchos de ellos $\mathrm{y}$, efectivamente, el nacimiento de un menor número de hijos por mujer debido a rupturas temporales o definitivas de la unión; todo esto puede, a su vez, ser diferencial entre generaciones.

Hecha la salvedad relativa a la cohorte de mujeres que se unió antes de los 15 años, la confirmación de que se trata de una población que se encuentra en un régimen de fecundidad natural, surge del hecho que, independientemente de la edad al contraer la primera unión, las tasas de fecundidad, para un mismo grupo de edades, son muy similares de una cohorte a otra (gráfica 2). Es decir, que la fecundidad de estas mujeres

Cuadro 3

TASAS DE FECUNDIDAD POR GRUPOS DE EDADES PARA 1000 MUJERES UNIDAS ENTRE LOS 15 Y 19 AÑOS DE EDAD

\begin{tabular}{lcccccccc}
\hline & \multicolumn{8}{c}{ Edad al tener 105 hijos nacidos vivos } \\
\cline { 2 - 7 } & $15-19$ & $20-24$ & $25-29$ & $30-34$ & $35-39$ & $40-44$ & $45-49$ & $\begin{array}{l}\text { Descen } \\
\text { dencia }\end{array}$ \\
\hline $\begin{array}{l}\text { MEXICO } \\
\text { (FERU-1970) }\end{array}$ & 361 & 431 & 369 & 335 & 215 & 122 & 21 & 8.48 \\
$\begin{array}{l}\text { FRANCIA } \\
\text { (Crulai 1674-1742) }\end{array}$ & 324 & 466 & 405 & 378 & 209 & 140 & 0 & 8.44 \\
$\begin{array}{l}\text { GRAN BRETANA } \\
\text { (Alredecor de 1920) }\end{array}$ & - & 320 & 165 & 90 & 50 & 20 & 0 & 3.20 \\
\hline
\end{tabular}

Fuentes: Cuadro 2 y L. Henry, Démographie: Analyse et Modéles. Larousse, Paris 1972 , pp. 105 y 122 .

está ligada a la capacidad reproductiva que poseen en función de su edad. Si se tratara de una población que ejerce un control efectivo sobre sus nacimientos, la mayor duración de unión implicaría diferencias en los niveles de la fecundidad de dos mujeres de la misma edad pero que se unieron en diferente momento.

En la gráfica 3 (datos cuadro 3 ) se comparan las tasas de fecundidad de las mujeres de la encuesta FERU unidas entre los 15 y 19 años de edad, por una parte, con las tasas correspondientes a un cohorte de mujeres de Crulai (Francia) que se casaron en esas mismas edades (entre los años 1674 y 1742) y que mantuvieron un régimen de fecundidad natural. La semejanza en la forma de las curvas, en los niveles de las tasas de fecundidad e incluso en la descendencia completa es evidente, especialmente si se les compara a su vez, con la curva correspondiente a las tasas de mujeres unidas aproximadamente a las mismas edades ( 20 años) en Gran Bretaña alrededor de 1920 pero que limitaban sus nacimientos. 


\section{Gráfica 3}

TASAS DE FECUNDIDAD POR GRUPOS DE EDADES PARA MUJERES QUE SE UNIERON ENTRE LOS 15 Y 19 AÑOS

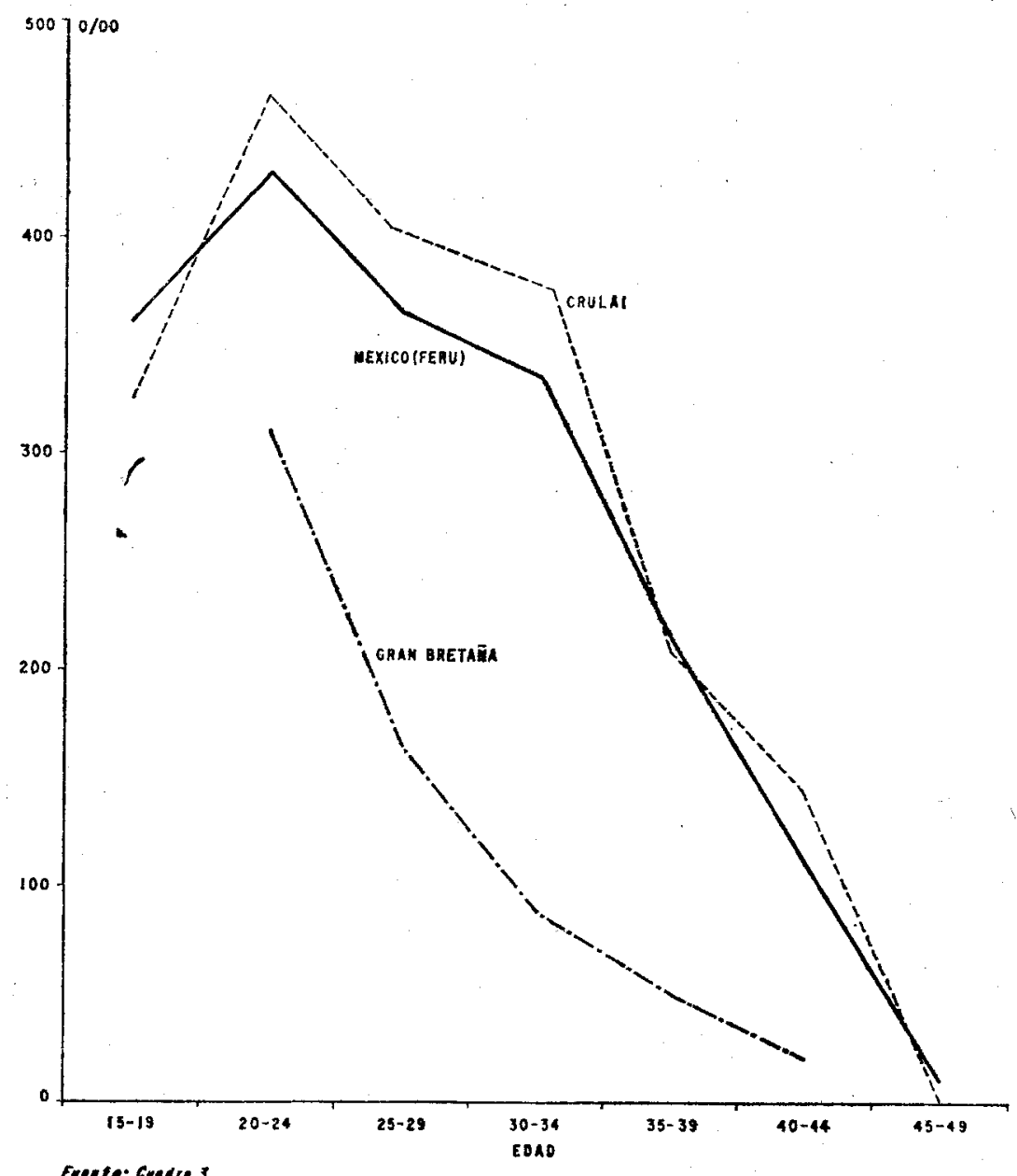

IV. Probabilidad de CRecimiento de LAS Familias

En un grupo donde las mujeres no ejercen ningún control voluntario sobre su número de hijos, la fecundidad que tengan las mujeres unidas se verá sólo limitada por los factores biológicos y por las costumbres que posean en relación con la exposición de la mujer al riesgo de concebir.

En una población que controla estas limitaciones "tradicionales", se 
suman las derivadas del uso de métodos más o menos efectivos destinados a impedir el nacimiento de hijos vivos, con la consecuente disminución de la probabilidad de encontrar mujeres con muchos hijos.

Con los datos relativos al número de hijos nacidos vivos de orden "i" clasificados por edad de la madre a la primera unión, se calcularon las probabilidades de crecimiento de las familias, correspondientes a las mujeres "alguna vez unidas" de 35 a 49 años, en la Encuesta de Fecundidad Rural de México. Estas probabilidades no responden exactamente a la definición de la probabilidad de crecimiento de las familias porque, por un lado, no todas las mujeres han completado la constitución de su descenden-

\section{Cuadro 4}

Probabilidades de CRecimiento de las familias, SEgún edad de la mujer a la primera unión, en diversos países

\begin{tabular}{|c|c|c|c|c|c|c|c|c|c|c|c|}
\hline & & & & & a & & & & & & \\
\hline & $a_{0}$ & $a_{1}$ & $a_{2}$ & $a_{3}$ & $a_{4}$ & $a_{5}$ & $a_{6}$ & $a_{7}$ & $a_{8}$ & $a_{9}$ & $a_{10}$ \\
\hline $\begin{array}{l}\text { MEXICO } 1 / \\
(\text { FERU-1970) }\end{array}$ & 0.982 & 0.982 & 0.977 & 0.974 & 0.961 & 0.954 & 0.904 & 0.885 & 0.757 & 0.758 & 0.674 \\
\hline $\begin{array}{l}\text { ARGELIA } 2 / \\
(\text { EnC. } 1972)\end{array}$ & 0.937 & 0.981 & 0.976 & 0.964 & 0.967 & 0.939 & 0.945 & 0.864 & 0.831 & 0.690 & 0.626 \\
\hline $\begin{array}{l}\text { ESPANA 3/ } \\
\text { (Censo 1950) }\end{array}$ & 0.9 .10 & 0.882. & 0.763 & 0.755 & & & & & & & \\
\hline $\begin{array}{l}\text { NORUEGA } 4 / \\
(1928-1930)\end{array}$ & 0.966 & 0.841 & 0.702 & 0.653 & 0.597 & 0.625 & 0.597 & 0.623 & & & \\
\hline
\end{tabular}

1 Mujeres "alguna vez unidas" de 35 a 49 años de la Encuesta de Fecundidad Rural (FERU), unidas entre los 15 y 19 años.

2 Etude Statistique Nationale de la Population: Résultats de l'enquête de fecondité. Argelia 1972. Gráfica 29, p. 78 (mujeres de 45 a 54 años unidas entre los 18 y 20 años).

${ }_{3}$ Leguina, J. Fundamentos de Demografia, Siglo XXI. España 1973, p. 251 (mujeres de 42 años o más que se casaron alrededor de los 18 años).

4 Henry, L., Demographie: Analyse et Modèles. Larousse, París, 1972, p. 127 (casadas a los 20-21 años entre 1928-1930).

cia (mujeres de 35 a 49 años en lugar de tomar sólo mujeres de 50 años); y por otro, se incluyen mujeres cuyas uniones se han visto interrumpidas en forma temporal o definitiva (mujeres con más de una unión, separadas, viudas y divorciadas), de modo que no todas han estado igualmente expuestas al riesgo de concebir.

En el cuadro 4 se han comparado las probabilidades de crecimiento de las familias en México (Encuesta de Fecundidad Rural), Argelia, España y Noruega. La elección de estos países reside en el afán de mostrar las diferencias que presentan estas probabilidades en países con niveles altos de fecundidad (México y Argelia) y en aquéllos con niveles bajos o relativamente bajos (Noruega, España).

En México, para el grupo 15-19 años de edad en la primera unión, la 
probabilidad de tener un segundo hijo es la misma que la de tener por lo menos un hijo ( 982 por mil). La probabilidad de tener un octavo y un noveno hijo habiendo tenido un séptimo y un octavo hijo respectivamente, no son muy diferentes entre sí ( 757 y 758 por mil). El descenso efectivo comienza a partir de $a_{8}$, o sea de la probabilidad de tener un noveno hijo habiendo tenido al menos 8 .

\section{Gráfica 4}

Probabilidad de CRecimiento de las familias, según Edad DE LA MUJER EN LA PRIMERA UNIÓN

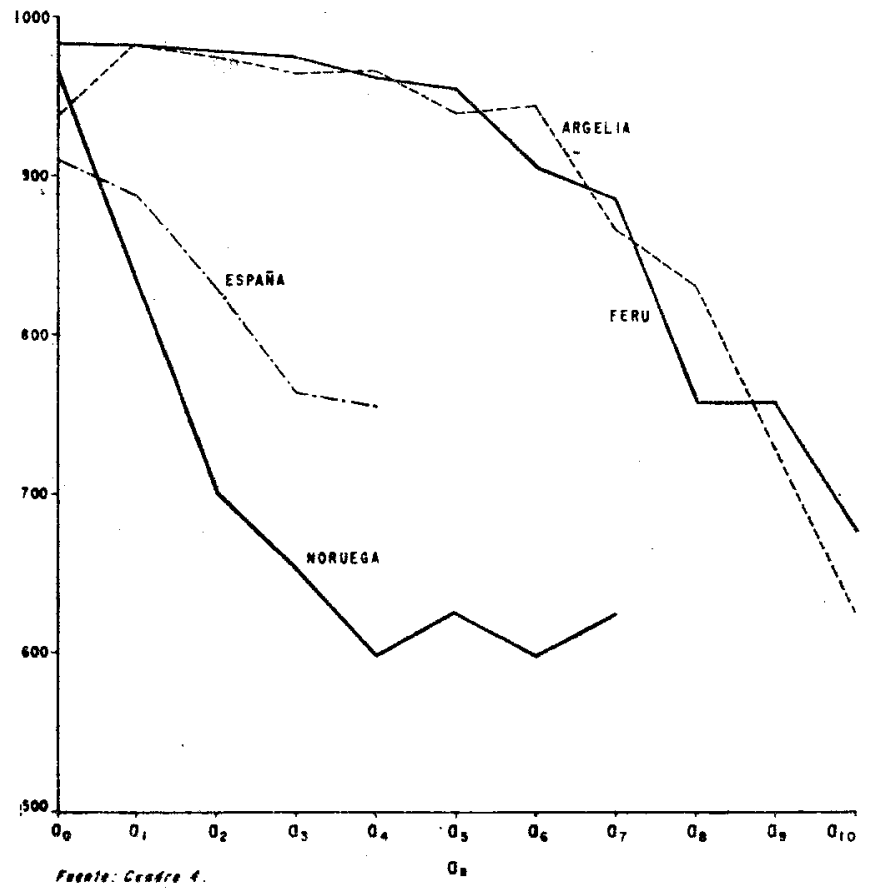

El complemento de 1000 de las probabilidades de tener al menos un hijo nos está indicando, que el número de mujeres (parejas) estériles desde el comienzo de la unión es menor (17 por mil en el caso de las mujeres unidas entre los 15 y 19 años) que el observado en otras poblaciones no malthusianas ( 35 por mil para mujeres casadas antes de cumplir 20 años).

La semejanza entre el nivel de probabilidad de crecimiento de las familias de México y Argelia, en contraste con aquéllos que presentan Es-

‘ R. Pressat, L'analyse Demographie. París, P.V.F., 2a. ed., 1969, p. 193, cuadro 47. 
paña y Noruega, apoya la conclusión anticipada en este trabajo, de que la población rural y semiurbana de México no utiliza métodos anticonceptivos para controlar su fecundidad.

Mientras la probabilidad de tener al menos 5 hijos en México o en Argelia es de 883 y 836 , en Noruega sólo alcanza 222 y en España, que posee una fecundidad algo más elevada, es de 383. Estas diferencias de niveles están haciendo evidente que en los países europeos seleccionados, una vez alcanzada la dimensión de familia deseada se recurre a métodos de control de los nacimientos. En la gráfica 4 se puede apreciar cómo estas diferencias se traducen en curvas cóncavas o convexas según se trate de países con alta o baja fecundidad.

Los tres tipos de mediciones efectuadas, con las limitaciones que cada una de ellas pueda tener, confirman las hipótesis sobre la ausencia de un control efectivo de la fecundidad entre las mujeres que vivían en las áreas rurales de México, en la década del sesenta. 